\title{
Reducción de la mortalidad materna en el Perú. ¿Por dónde empezar?
}

\author{
BRUNO BENAVIDES \\ Jefe del Equipo de Asistencia Técnica del Proyecto 2000
}

"Un profundo, oscuro flujo continuo de mortalidad... ¿por cuánto tiempo continuará este sacrificio?" Más de siglo y medio ha transcurrido desde que William Farr (') formuló esta pregunta relativa a las muertes maternas en Inglaterra. Tristemente, esta pregunta continúa siendo relevante en el mundo globalizado y tecnológico-de las computadoras, estación espacial, teléfonos celulares y corazones artificiales-del siglo XXI.

\section{Pobreza y mortalidad materna}

La mortalidad materna es uno de los indicadores sanitarios que con más tidelidad expresa la iniquidad y la exclusión social. Más de medio millón de muertes maternas se producen anualmente en el mundo. El $99 \%$ sucede en los países en desarrollo. Vivir en condiciones de pobreza limita a las nujeres el acceso a los recursos cconómicos, sociales y a la educación básica. De cse modo, disminuye su capacidad de tomar decisiones informadas en salud y nutrición. La mortalidad materna expresa, además, la iniquidad de género para la roma de decisiones, para los derechos fundamentales y los servicios sociales.

La mortalidad materna afecta principalmente a las mujeres pobres, excluidas de los servicios de educación y salud, quienes carecen de poder para decidir aún en el seno de sus propias familias. Detrás de estas muertes se agazapa la

\section{Correspondencia:}

Dr. Bruno Benavides

Jefe del Equipo de Asistencia Técnica. Proyecu 2000 Ministerio de Salud

Av. Salaverry Cdra. $8 \mathrm{~s} / \mathrm{n}$. Lima 11, Perú

E-mail:bbenavides@viabcp.com poca capacidad de negociación y autodeterminación de las mujeres $\left({ }^{2}\right)$.

Pero no sólo expresa la pobreza, es también causa de la misma. En por lo menos la cuarta parte de hogares con varones como jefes de familia, las mujeres aportan con más de la mitad de los ingresos $\left({ }^{3}\right)$. La muerte de esa mujer profundiza la pobreza familiar $\left({ }^{4}\right)$. Es aún peor cuando la mujer que muere es cabeza de familia. En Perú, esto sucede en $20 \%$ de hogares $\left(^{5}\right)$.

El costo social de la mortalidad materna se expresa en la consecuente desorganización familiar. Los niños ven incrementarse el riesgo del abandono, con sus consecuencias de desnutrición y deficiente educación. Los niños de un hogar que pierde a su madre se profundizan en el círculo vicioso de la pobreza. Más aun, se ha descrito que sólo uno de cada diez recién nacidos que perdieron a su madre alcanza a cumplir su primer año $\left({ }^{4}\right)$.

\section{¿Por qué priorizar la reducción de la morta- lidad materna?}

No dejan de escucharsc voces que preguntan ¿por qué es tan importante la muerte materna, cuando ocurrén muchas más muertes por orras causas, por ejemplo, accidentes de tránsito? Esta pregunta aparenta una preocupación epidemiológica, pcro en realidad pone de manifiesto un dilema ético que dcbe ser resuelto por la sociedad. Debemos preguntarnos: ¿hasta qué punto nuestra organización e instituciones sociales son capaces de garantizar la vida de las mujeres y asegurar que eventos absolutamente naturales $c$ indispensables para la supervivencia de la especie humana, como la maternidad, se desarrollen de manera voluntaria, saludable y segura? 
Durante las dos últimas décadas hemos sido testigos de una significativa reducción de la mortalidad in rantil, principalmente por la disponibilidad y uso masivo de tecnologías de bajo costo y complejidad, como las vacunas y la tcrapia de rehidratación oral. Para el caso de la mortalidad materna, aún no existen tecnologías comparables. No hay una vacuna ni una "bolsita salvadora" contra la hemorragia. Para reducirla, es preciso que existan servicios de salud que oferten cuidados obstćtricos esenciales de calidad y que éstos sean utilizados por las gestantes. Para que se produzca cl uso de servicios se requicre de vías de comunicación modernas, medios de transporte, usuarias educadas y con autodeterminación para optar por los servicios de salud, especialmente al momento del parto. Los establecimientos de salud deben organizarse y ofertar scrvicios culturalmente accptables para el grupo étnico específico al cual sirven. Además, estos servicios deben estar adecuadamente linanciados, evitando lá exclusión de los más pobres.

En suma, se requicre de desarrollo social y humano. La mortalidad materna está siendo crecientemente considerada como el más importante indicador de desarrollo en sustitución de indicadores cconómicos $\left({ }^{6}\right)$, un indicador muy sensible del progreso de los pueblos.

\section{Causas y soluciones}

¿Cuál es el misterio de las complicaciones que matan a las mujeres gestantes? Ninguno. Estas complicaciones son conocidas desde hace mucho ticmpo y, sorprendentemente, su distribución es bastantc parecida entro países desarrollados y aquellos en desarrollo $\left({ }^{7}\right)$.

La hemorragia, la sepsis, el aborto complicado y el parto obstruido constituyen $90 \%$ de las causas directas de las mucrtes maternas $\left({ }^{8}\right)$. Esto no ha cambiado desde que se iniciara la recopilación de información y análisis de este problema, hace más de treinta años. Entonces, cabe preguntarse por qué no logramos reducir significativamente la razón de mortalidad materna, a pesar que conoccmos las causas clínicas, comprendemos la epidemiología del problema, contamos con las herramientas tecnicoclínicas para resolverlo y se ha implementado diversas iniciativas para conseguirlo.

La ausencia de resultados positivos cn esta guerra por hacer de la maternidad un cvento voluntario, saludable y seguro no puede ser atribuida a errores circunstanciales, a gestiones transiforias, a iniciativas erróneas de corto plazo, ni a problemas circunscritos a determinadas regiones del país. Décadas de ausencia de resultados positivos hablan de más de cincuenta mil mujeres muertas y del consiguiente iracaso y irustración de varias generaciones de profesionales y funcionarios, cn los niveles locales, regionales y nacional.

La diferencia en la evolución de la mortalidad materna en Perú y otros países en desarro110 con los países desarrollados es que, en los últimos, es prácticamente nula la mortalidad por aborto complicado-debido a la disponibilidad de scrvicios de aborto seguro legalmente respaldados- y la muerte por parto obstruido casi no existc gracias al acceso a la cesárea.

Los países desarrollados han reducido sensiblemente la mortalidad materna, principalmente porque hoy en día se cucnta con el conocimiento y la tecnología capaces de lograrlo. La enorme diferencia en la mortalidad materna entre los países desarrollados y aquellos en desarrollo, se explica principalmentc por las diferencias de acceso a servicios de salud, capaces de ofertar cuidados obstétricos csenciales de calidad. Este mismo razonamiento nos permite explicar las difcrencias observadas dentro de los países en desarrollo, entre zonas urbanas y rurales, y entre ricos y pobres.

Es preciso comprender el mccanismo por el cual el desarrollo social favorece cl logro de un objetivo sanitario. Para el efccto, puede postularse al menos tres modelos:

a) El comporramiento como prevención. Se créd la infraestructura que, sumada a cambios en la distribución del poder y una mejor cducación de la población, respalda cambios de 
comportamiento que conducen directamente a evitar un daño. Por ejemplo, el consumo de agua potable evita infecciones intestinales, lo que a su vez reduce la mortalidad por las mismas.

b) La intervención sanitaria como prevención. Se pone a disposición de la población recursos sanitarios que, sumados a cambios en la distribución del poder y una mejor educación de la población, son intensamente utilizados y, en consecuencia, se previene el daño. Por ejemplo, la administración de vacunas previene la ocurrencia de enfermedades inmunoprevisibles y así se reduce la mortalidad por esta causa.

c) La intervención sanitaria como reparación. Se pone a disposición de la población recursos sanitarios que, sumados a cambios en la distribución del poder y una mejor educación de la población, son intensamentc utilizados, y entonces reparan el daño ya existente. Por ejemplo, la extracción manual de placenta o la histerectomía controlan la hemorragia y así se reduce la mortalidad materna.

Para reducir la mortalidad materna tenemos a nuestra disposición conocimiento y tecnología, que nos permiten organizar intervenciones sanitarias, tanto para prevenir como para curar el daño.

La intervención preventiva por excelencia es la planificación familiar. La primera condición para que ocurra una muerte materna es que la mujer esté gestando. Para mujeres portadoras de antecedentes obstétricos de riesgo, el acceso y uso con consentimiento informado de métodos anticonceptivos elegidos por ellas puede ser la diferencia entre la vida y la mucrte. La planificación familiar es también la mejor opción en el caso de mujeres, particularmente adolescentes sexualmente activas, quienes por el momento no desean gestar. Una gestación no deseada es una invitación al aborto. Dado que éste está penalizado, muchas veces se ofrece en condiciones de clandestinidad e insalubridad, siendo la puerta hacia el oscuro y triste final de una vida. Hoy en día, mujeres víctimas de una cobarde violación pueden optar por la anticoncepción oral de emergencia dentro de las 72 horas posteriores a la violación, antes que recurrir al aborto. La planificación familiar salva vidas.

Una forma indirecta de comprobar el efecto de la planificación familiar sobre la mortalidad es analizar la razón de mortalidad materna reportada por ENDES 1996 y 2000. Esta razón puede expresarse de dos maneras:

a) Utilizando como denominador a la población de recién nacidos vivos. Permite una aproximación al número de gestantes; por tanto, se considera como población expuesta al riesgo a quienes ya se encuentran gestando. De esa manera, esta razón mide la efectividad de las intervenciones obstétricas.

b) Utilizando como denominador a la población de mujeres en edad fértil. En este caso, a la población de gestantes se agrega aquella de mujeres en edad fértil que no se encuentran gestando. Esta razón mide tanto la efectividad de las intervenciones obstétricas como aquella de las intervenciones de planificación familiar.

Al comparar las cifras de ambas razones reportadas para el año 2000 con aquellas reportadas para 1996, se observa que la razón por nacidos vivos se habría reducido en $30,2 \%$, mientras que la razón por mujeres en edad fértil se habría reducido en $42,1 \%$. Esta diferencia puede ser atribuida a las intervenciones en planifi cación familiar.

En el caso de las complicaciones de la gestación, parto y puerperio, el desarrollo social no las previene. Su efecto es mediado por la existencia y uso de servicios de salud que oferten cuidados obstétricos esenciales de calidad. Mujeres con elevados ingresos económicos, buena nutrición y educación no tienen garantizada ni la ausencia de complicaciones durante la gestación y el parto, ni una solución de las mismas. Esto fue comprobado por un estudio en los Estados Unidos, en 1983, en el cual se registró una razón de mortalidad materna de 872 por 
100000 nacidos vivos en mujeres de posición social elevada, en una comunidad musulmana fundamentalista que no aprobaba la atención obstétrica ni la asistencia médica moderna $\left({ }^{9}\right)$.

Cobra plena vigencia las conclusiones de un reporte de la Organización Panamericana de la Salud $\left({ }^{10}\right)$, las que sostuvieron que la mortalidad materna guarda una relación directa con la tasa global de fecundidad, y una relación inversa con la prevalencia de uso de anticonceptivos, la cobertura de atención prenatal y la cobertura de atención institucional del parto.

\section{La mortalidad materna en el Perú \\ Situación actual}

De acuerdo a la ENDES 2000 , la razón de mortalidad materna es de 185 muertes por 100 000 nacidos vivos. En números absolutos significa aproximadamente 1258 muertes anuales. Si aplicamos estimaciones internacionales (11), las complicaciones de la gestación y el parto que produjcron estas muertes son, además, responsables de aproximadamente seis mil muertes neonatales precoces (durante la primera semana de vida) y doce mil natimuertos.

La cifra estimada de mortalidad materna continúa siendo muy alta. Es preciso conocer que la metodología de medición de este indicador utilizado por el INEI tiene una imprecisión de $\pm 20 \%\left({ }^{12}\right)$. Esto significa que, en realidad, la razón de mortalidad materna podría ser cualquier valor ubicado entre 148 y 222 por $100 \mathrm{mil}$ nacidos vivos, es decir, entre 1006 y 1510 muertcs anuales.

La notificación de muertes matcrnas ha mejorado mucho en el Ministerio de Salud, desde que la Oficina General de Epidemiología ha implementado su vigilancia en todas las DISA del país. Sin embargo, en el año 2000 sólo se notitícó 655 muertes $\left({ }^{13}\right)$, lo que representaría un nivel de subregistro de aproximadamente $48 \%$. Lo más probable es que el subregistro corresponda a muertes Tipo IV, es decir, aquellas ocurridas en gestantes sin atención prenatal y con parto domiciliario, quienes no tuvicron contacto alguno con el sistema de salud durante la gestación ni durante el parto-puerperio.

Lamentablemente, no existe una forma confiable de estimar el subregistro de muertes maternas en cada DISA y, por ello, no puede conocerse las cifras reales del total ni de las muertes Tipo IV. Pese a ello, la información proporcionada es valiosa y permite calegorizar a las DISA según el número de muertes maternas notificadas (Tabla 1).

Tabla 1.- Distribución de muertes matemas notificadas según departamentos.

\begin{tabular}{cc}
\hline Departamentos & $\begin{array}{c}\text { Número de } \\
\text { muertes maternas } \\
\text { notificadas }\end{array}$ \\
\hline
\end{tabular}

Tacna, Tumbes, Madre de Dios, Moquegua, Ica, Pasco, Ucayali, Arequipa $<20$

Apurimac, Amazonas, Huancavelica, Lambayeque, San Martin, Ayacucho, Ancash, Junín, Loreto, Huánuco $20-39$ Piura, La Libertad, Lima, Cusco $40-59$ Cajamarca, Puno $60-80$

\section{Muerte materna y uso de servicios}

Dada la importancia ya discutida del acceso a la atención prenatal y a la atención insticucional del parto, es preciso conocer la relación entre el uso de dichos servicios y las muertes maternas.

Utilizando datos de la ENDES 2000, y considerando que la situación óptima es que una gestante reciba atcnción prenatal y atienda su parto en un establecimiento de salud, se calculó la razón de probabilidades (odds ratio) de la muerte tipo I contra los demás tipos de muerte materna (Tabla 2). Para ello se utilizó como denominador de cada celda al número de gestantes estimadas por el Ministerio de Salud para el año 2000, distribuidas según los hallazgos muestrales de ENDES 2000. Se asumió 
que las muertes no notificadas ocurrieron en el domicilio y que habían tenido una exposición al servicio de atención prenatal similar al registrado por las muertes maternas en domicilio que sí fueron notificadas.

Tabla 2.- Riesgo de muerte materna según uso de servicios.

\begin{tabular}{lcc}
\hline Tipo de muerte & $\begin{array}{c}\text { Razón de probabilidades } \\
\text { (odds ratio) }\end{array}$ \\
\hline Tipo I $\quad \begin{array}{l}\text { (parto institucional } \\
\text { con atención prenatal) }\end{array}$ & 1,00 \\
Tipo II $\quad \begin{array}{l}\text { (parto domiciliario } \\
\text { con atención prenatal) }\end{array}$ & 9,17 \\
Tipo III $\quad \begin{array}{l}\text { (parto institucional } \\
\text { sin atención prenatal) }\end{array}$ & 11,99 \\
Tipo IV $\quad \begin{array}{l}\text { (parto domiciliario } \\
\text { sin atención prenatal) }\end{array}$ & 5,79 \\
\hline
\end{tabular}

Estos resultados confirman que las mujeres que no reciben atención prenatal y dan a luz en su domicilio tienen 6 veces más probabilidades de morir que aquellas que reciben atención prenatal y dan a luz en un establecimiento de salud. El elcvado riesgo observado en las muertes tipo III puede atribuirse a tres posibilidades no excluyentes:

- Que las gestantes que no tuvieron contacto con los servicios, hacen uso de ellos recién cuando una complicación amenaza su vida, siendo muchas veces muy tarde.

- Que los servicios de salud no están preparados para resolver emergencias totalmente imprevistas.

- Que las gestantes buscaron ayuda en servicios de salud de baja complejidad, los cuales no ofrecen cuidados obstétricos esenciales.

Es útil analizar lo que está sucediendo con las muertes tipo I, debido a que expresa la capacidad de los servicios de salud para evitar muertes maternas en el mejor escenario, es de- cir, cuando las gestantes tienen atención prenatal y la atención del parto es institucional. En el caso de las muertes tipo I, la cifra notificada por las DISA es asumida como real, debido a que muy difícilmente se dejaría de notificar una muerte ocurrida en el establecimiento de salud.

El cálculo de la tasa de mortalidad materna (muertes notificadas/gestantes estimadas) para el tipo I arroja un promedio nacional de 38 muertes por cada 100000 gestantes. Como ya es conocido, debido a la gran heterogeneidad del país, los promedios ocultan brechas existentes entre las regiones, tal como se puede observar en la Tabla 3.

Tabla 3.- Tasas de mortalidad materna lipo I según departamento (muertes maternas notificadas/gestantes estimadas).

\begin{tabular}{lc}
\hline Departamento & $\begin{array}{c}\text { Tasa de mortalidad } \\
\text { materna tipo I }\end{array}$ \\
\hline Tacna & 0 \\
Lima & 11 \\
Tumbes & 19 \\
Ica & 25 \\
Arequipa & 29 \\
Ucayali & 31 \\
Moquegua & 31 \\
Cusco & 33 \\
Lambayeque & 36 \\
Promedio Nacional & 38 \\
Madre de Dios & 42 \\
Ancash & 42 \\
Piura & 52 \\
Junín & 57 \\
La Libertad & 57 \\
Apurímac & 67 \\
Ayacucho & 74 \\
Pasco & 79 \\
Huánuco & 83 \\
Loreto & 94 \\
Amazonas & 105 \\
Puno & 110 \\
San Martin & 112 \\
Huancavelica & 124 \\
Cajamarca & 134 \\
\hline
\end{tabular}


Del estudio de la Tabla 3, puede deducirse que, si el camino para reducir la mortalidad materna es incrementar el acceso a cuidados obstétricos de calidad, tenemos mucho por hacer, pues aún en el escenario óptimo (atención prenatal + parto institucional) la tasa de mortalidad materna es baja $(<20)$ en sólo 3 DISA, moderada (20-59) en 11 DISA y alta (60-149) en 10 DISA.

Mejorar la calidad de los cuidados obstétricos esenciales debe ser una de las prioridades nacionales en la lucha contra la mortalidad materna. Aunque la magnitud de la tarea es enorme, vale la pena comprender la forma cómo la población utiliza los servicios de salud, con la finalidad de poder focalizar las acciones siguiendo dicho pairón de uso. Según resultados del $2^{\circ}$ Censo de Infraestructura y Recursos del Sector Salud $\left({ }^{14}\right)$, $77 \%$ ( 158600 casos) de los partos institucionales y $97 \%$ (28057 casos) de las cesáreas ocurren en los hospitales, los cuales representan 2,2\% (139 casos) del total de establecimientos del país (6214 casos). Si este ejercicio se realizara en cada una de las DISA, probablemente se concluirá que el número de establecimientos que concentran $80 \%$ de los partos institucionales es pequeño y con mucha seguridad coinciden con los hospitales departamentales y los establecimientos cabecera de red. La suma de estos establecimientos a nivel nacional es aproximadamente trescientos cincuenta.

En otras palabras, realizando una intervención intensiva en un número relativamente pequeño de establecimientos, es posible mejorar significativamente y a corto plazo la calidad de los servicios de atención de partos ofertados a la inmensa mayoría de sus usuarias, impactando directamente sobre un porcentaje significativo de las muertes maternas, e indirectamente al elevar la confianza de la población sobre la capacidad del sistema para garantizar la calidad de los servicios $y$, consecuentemente, la vida de sus pacientes. Esto tiene sentido, toda vez que la complejidad de los cuidados obstétricos esenciales no puede ser ofertada, con la calidad debida, en centros y puestos de salud de baja complejidad.

\section{Utilidad de cstrategias e intervenciones co- múnmente utilizadas}

\section{Enfoque de riesgo}

Cuando en los años ' 60 -' 70 se introdujo el enfoque de riesgo en la salud pública, había certeza que para cada daño existía un grupo de factores predictivos. Al conocerse los factores predictivos, era posible seleccionar a los individuos portadores de los mismos, quienes recibirían cuidados especiales para evitar o atender oportunamente el daño.

La aplicación mecánica de este enfoque condujo a la elaboración de listas de factores supuestamente predictivos de las complicaciones que matan a las mujeres gestantes. Hoy en día es común encontrar en nuestros hospitales los denominados "consultorios de alto riesgo". Estudios recientes han demostrado que, en realidad, la mayoría de mujeres con "factores de riesgo" no presentan complicaciones que pongan en peligro su vida y que la mayor parte de las defunciones asociadas con la gestación se producen como consecuencia de complicaciones imprevistas, es decir, las que aparecen entre las mujeres no identificadas como "de riesgo" $\left({ }^{15.16}\right)$.

El concepto vigente asume que todas las mujcres gestantes están en riesgo de morir por complicacioncs obstétricas $\left({ }^{17}\right)$.

\section{Atención prenatal}

Entonces surge la pregunta, si con la tecnología actualmente disponible no es posible predecir quiénes se verán alectadas por alguna complicación eventualmente letal, ¿cuál es el valor de la arención prenatal? Responder a csta pregunta es ingresar a un debate que se remonta a las primeras décadas del siglo pasado $\left({ }^{18}\right)$. Maine $\left({ }^{19}\right)$ ha hecho explícitas sus dudas y críticas sobre el valor de la atención prenatal, concluyendo que una valoración de los mismos depende de cóno, bajo qué circunstancias, qué elementos los constituyen y revisando si merecen la prioridad casi exclusiva que hoy tienen en muchos países. 
Ciertamente el poder de la atención prenatal ha sido sobredimensionado. Cuando en un taller con gerentes de servicios de salud de Ayacucho se preguntó cómo podía explicarse la diferencia de coberturas entre las consultas prenatales y la atención de parto institucional, el jefe de un hospital rural mencionó que muchas mujeres acuden a la consulta prenatal simplemente para saber cómo va su gestación. Si hasta ese momento no se presenta ningún problema, entonces consideran que no existe suficiente justificación para continuar asistiendo a sus consultas prenatales ni para que el parto deba ser institucional. Esta situación sc agrava si el mensaje del proveedor de salud confiere poder curativo a la atención prenatal, al afirmar que acudir a cuatro consultas asegura un final feliz de la gestación.

Indudablemente, durante la atención prenatal, aunque limitadamentc, es posible identificar condiciones polencialmentc de riesgo, como:

- Gestantes grandes multíparas, o portadoras de gestaciones múlriples o polihidramnios, condiciones que favorecen la cventual presentación de atonía uterina en el pucrperio inmediato, una de las principales causas de hemorragia uterina.

- Gestantes con antecedentes de cesáreas previas o con abortos a repetición.

- Gestantes afectadas con anemia o infecciones de transmisión sexual.

- Si se cuenta con diagnóstico por imágencs, también puede identificarse la presencia de placenta previa.

- Es también factible, en muchos casos, identificar signos y síntomas de hipertensión inducida por el embarazo.

- Igualmente puede monitorizarse el crecimiento fetal y diagnosticar retardo de su crecimiento. Con ultrasonografía, también puedc detectarse malformaciones congénitas y cuadros de abdomen agudo en el feto $\left({ }^{20}\right)$.
Todos estos diagnósticos deberían permitir la toma de decisiones para instaurar tratamientos específicos y, principalmente, persuadir a la gestante y sus familiares sobre la imperiosa necesidad que el parto ocurra en un establecimiento con capacidad de ofertar cuidados obstétricos esenciales en caso de requerirse. Sin embargo, la atención prenatal está marcada por una lógica productivista, que privilegia la consecución de metas cuantitativas $y$, en muchos casos, no se diagnostica o no se hace el seguimiento necesario.

Existe evidencia que la atención prenatal tendría urilidad para la ocurrencia de pártos seguros. Un estudio en la India $\left({ }^{21}\right)$ ha revelado que, aquellas mujeres con un relativamente elevado nivel de atención prenatal tuvieron casi cuatro veces más posibilidades de ser asistidas durante el parto por personal competente, condición indispensable para recibir cuidados obstétricos esenciales en caso de requerirse.

Del mismo modo, un análisis reciente de la ENDES 2000 muestra que el riesgo de muerte materna era $9,82(8,75-11,02)$ veces mayor en ausencia de atención prenatal. Aparentemente, el mecanismo explicativo es el mismo observado en la India. Más allá del valor predictivo de la atención prenatal, ésta juega un papel importante en lograr que las gestantes elijan el parto institucional y, de esta mancra, adquieran mayor protección en caso de aparecer complicaciones eventualmente mortales. Esto se verifica cuando, de la información recogida por ENDES 2000 , se observa que en ausencia de atención prenatal el riesgo de parto domiciliario fue 8,88 $(8,75-9,03)$ veces mayor.

\section{Atención institucional del parto y capacitación de parteras tradicionales}

Por mucho tiempo, la política de salud ha sido ecléctica respecto a la promoción del parto institucional. Evidencias de resistencia de las mujeres, principalmente rurales, a utilizar este servicio han puesto continuamente sobre la mesa la discusión de corregir esta situación 
a través de la capacitación de las parteras tradicionales.

Con el conocimiento y la experiencia acumulada durante las últimas décadas, puede afirmarse que las parteras tradicionales aportan de dos maneras a la reducción de la mortalidad materna:

a) La capacitación en técnicas de parto limpio puede ser útil para disminuir el riesgo de infecciones puerperales.

b) La partera tradicional es una aliada estratégica del servicio de salud. Dado que es un referente importante para la población, ella puede cumplir una función de bisagra intercultural.

Pero, respecto de otras causas de muerte materna -como hemorragias y eclampsia-, resultaría injusto que descargáramos sobre estas personas la responsabilidad por vidas humanas, cuando no cuentan con los conocimientos, habilidades, y equipamiento requerido para proporcionar cuidados obstétricos esenciales. Recientes estudios $\left({ }^{22}\right)$ sugieren que esta alianza puede operativizarse cuando los servicios incorporan técnicas tradicionales de atención de parto. La parturienta tiene la opción de elegir entre el parto atendido con técnicas tradicionales o con técnicas occidentales. Si la elección es el parto tradicional, éste sería atendido por parteras tradicionales, con el respaldo de profesionales que actuarían ante la presencia de alguna complicación. En algunos lugares del país, los profcsionales se han capacitado en el uso de técnicas tradicionales.

Es preciso ser conscientes que en el Perú nos ubicamos en plena transición, desde el parto atendido por parteras tradicionales hasta el parto institucional, habiéndose reportado una consiscente reducción del porcentaje de los partos atendidos por parteras tradicionales desde 1986 hasta la fecha (ENDES 1986, 1991, 1996, 2000).

Es preciso acotar que, al igual que en el caso de la atención prenatal, el parto atendido institucionalmente tampoco protege, por sí mismo, de las complicaciones y la nuerte de muje- res. Lo que sucede es que, al estar en un ambiente sanitario, se acrecientan las posibilidades de acceso oportuno a los cuidados obstétricos esenciales.

Dado que muchos de estos cuidados requieren de mayor complejidad que aquella que existe en los puestos y la mayoría de los centros de salud, es preciso no sólo captar más parturientas al parto institucional, sino garantizar el funcionamiento adecuado de la red de servicios, para facilitar el acceso de las mujeres a aquellos establecimientos que oferten cuidados obstétricos de calidad. Esto supone un esfuerzo notable para que en todos los centros y puestos de salud se oferten cuidados obstétricos bási$\cos$, que permitan la estabilización y referencia inmediata de pacientes en caso de requerirlo.

En conclusión, es necesario acelerar la transición hacia la atención institucional del parto, incorporando a las parteras tradicionales y las técnicas tradicionales de atención del parto en el sistema de atención y fortaleciendo las capacidades de las redes de servicios para ofertar cuidados obstétricos básicos y esenciales y contar permanentemente con los medios para una referencia oportuna y adecuada de las pacientes que requieran cuidados obstétricos esenciales al establecimiento de la red encargado de ofertarlos con calidad.

\section{Casas de espera}

Ésta es una estrategia orientada a facilitar el acceso de las gestantes a cuidados obstétricos esenciales, reduciendo los riesgos derivados de la presentación inesperada de una complicación obstétrica en su hogar o en un establecimiento de nivel básico. Dado que no es posible que todas las gestantes utilicen esta facilidad, su uso debería priorizarse para aquellos casos en los cuales es posible detectar un antecedente obstétrico de riesgo. El número de casas de espera se ha incrementado notablemente en el páís en años recientes; lamentablemente, no se cuenta con información que permita evaluar su utilización y relación con la atención oportuna de emergencias obstétricas. 
Vigilancia y seguimiento de gestantes y puérperas en el ámbito comunitario

Para el desarrollo de esta labor es importante considerar que la pareja y el entorno familiar directo de la gestante deben estar necesariamente involucrados en la oferta de información y orientación acerca de los cuidados y riesgos durante el embarazo, parto y puerperio, por cuanto asumen un papel protagónico en la toma de decisiones para la búsqueda de atención en caso de urgencias. Por otro lado, el trabajo conjunto y articulado entre el establecimiento de salud y la comunidad organizada debe permitir complementar recursos y esfuerzos necesarios para salvar vidas, potenciándose la eficiencia y eficacia de la intervención.

La identificación de la totalidad de gestantes que forman parte de la población asignada al establecimiento es un requisito indispensable para implementar de manera adecuada una estrategia de promoción del cuidado de la salud. El registro inmediato de esta información en el radar de gcstantes pcrmite ubicar visualmente a las gestantes, tipificar su riesgo, hacer la programación de visitas domiciliarias y contar con la programación mensual de partos esperados.

En el caso de la gestante identificada, la visita domiciliaria tiene como propósito interesar y comprometer a la gestante y a su familia para acudir a la consulta prenatal, en tanto les informa acerca de los bencficios de la consulta prenatal y cl parto institucional. La visita domiciliaria a usuarias con control prenatal busca reforzar los mensajes de salud y apoyar a la familia para organizar y planificar el parto. De esta manera, se favorece la identiticación temprana de aquellos riesgos plausibles de predicción y el traslado oportuno y organizado de la gestante.

Contar con el inventario de los medios de comunicación y los recursos comunitarios disponibles en la jurisdicción del éstablecimiento de salud le permite al proveedor hacer un mejor uso de los mismos frente a situaciones de emergencia y evacuación. Esta información también es útil para la gestante y su familia al permicir- les identificar las rutas, las formas de apoyo y los medios más adecuados para la evacuación oportuna.

\section{El seguro materno infantil}

E1 Ministerio de Salud ha definido como una de sus prioridades la remoción de la barrera económica que limita el acceso de las mujeres a los servicios obstétricos esenciales. Quince departamentos del país han sido incorporados al seguro, el cual incluye el subsidio a traslados de emergencia en áreas rurales y periurbanas, consultas prenatales, hospitalización, atención del parto, cesárea y emergencias $\left({ }^{13}\right)$. Todavía no sc cuenta con reportes precisos sobre el efecto de esta iniciativa sobre la utilización de servicios, su relación con la atención de emergencias obstétricas y el impacto sobrc la mortalidad. Información preliminar permite apreciar que esta estrategia por sí sola no es suficiente para reducir la brecha entre la cobertura de atención prenatal y de acención institucional del parto, lo que ha motivado a los responsables del programa a revisar ocros factores que continúan limitando el acceso a los servicios, aún en ausencia de la barrera económica.

\section{Garantizando cuidados obstétricos de calidad}

Para garantizar los cuidados obstétricos esenciales debemos responder a la pregunta ¿qué es calidad de atención? De las múltiples definicioncs disponibles, la expericncia positiva del Proyccto 2000 permite concluir que debemos adoptar una postura integral, comprehensiva y simple. Podemos definir a un scrvicio de calidad como aquel que cumple las funciones para las que fuc creado. Pero, ¿quién define las funciones? En este punto también hemos aprendido que es necesario establecer un diálogo entre el Estado y la ciudadanía y, por tanto, es preciso incorporar su perspectiva en la formulación de las políticas públicas.

La frase arención con calidad y calidez implícitamente reduce la calidad a la eficacia tecnicoclínica. Para otros, la calidad cstá pre- 
dominantemente vinculada a la satisfacción del usuario, lo que a su vez dependería principalmente del trato recibido. Estas apreciaciones resultan parciales y responden a una perspectiva propia de la relación entre individuos y los servicios de salud; adicionalmente, en ambos casos se parte del supuesto de una relación ya establecida entre usuarios y prestadores $y$, por ello, la calidad aparece desvinculada de los no usuarios, quienes en caso de la mortalidad materna constituyen el grupo poblacional de mayor riesgo.

Un modelo que permite un diálogo nuido entre las perspectivas del Estado y de la ciudadanía es el desarrollado por Maxwell $\left({ }^{23}\right)$ para evaluación de servicios de salud. Desde esta perspectiva, a los aspectos propios de la relación entre individuos y prestadores, se agregan aquellos propios de la organización del sistema, con relación a la población. Su adaptación para la formulación de los cuidados obstétricos esenciales nos permite concluir que éstos deben ser ofertados garantizando que contengan los siguientes atributos de calidad:

1. Accesibilidad. Es necesario que existan recursos humanos competentes, así como equipamiento, medicamentos e insumos necesarios para proporcionar cuidados obstétricos esencialcs. Esto incluye contar con el presupuesto adecuado, un sistema logístico óptimo, un enorme esfuerzo de capacitación y actualización, así como proporcionar facilidades para que los profesionales puedan satisfacer necesidades propias de sus expectativas personales, familiares y profesionales.

Las gestantes y sus familiares deben estar debidamente informadas sobre cuándo y dónde deben buscar ayuda profesional. De este modo, cs factible reducir el primer retraso $\left({ }^{24}\right)$ hacia la iniciación oportuna del tratamiento requerido.

Adicionalmente, deberá asegurarse los medios que permitan a las gestantes llegar al establecimiento cabecera de red u hospital departamental; es decir, el resultado de vías de comunicación, medios de transporte y comunicación disponibles, y apropiados procedimientos de referencia de pacientes víctimas de alguna complicación que requiera cuidados obstétricos esenciales.

Simultáneamente, los servicios de salud deben articularse con el entorno sociocultural de las poblaciones a las que sirven. Un sistema de salud que brinda servicios que responden a la cultura occidental urbana, excluye automáticamente a los diversos grupos étnicos existentes en el país. La articulación es un enorme esfuerzo que recientemente viene sistematizándose y encontrando acogida en los niveles de decisión del Ministerio de Salud.

2. Equidad. Toda gestante que requiera cuidados obstétricos esenciales debe poder acceder a los mismos, sin que medie ninguna barrera de tipo económico que la limite. Medidas transitorias, como una adecuada focalización del subsidio público dirigida a las poblaciones más pobres o la futura universalización de seguros públicos, son indispensables para conseguir este requisito.

3. Efectividad. Todo establecimiento debe garantizar a sus usuarias que los cuidados obstétricos esenciales se brinden de acuerdo a protocolos estandarizados, actualizados, internacionalmente aceptados, y que sus equipos de salud sean competentes en su aplicación. Por tanto, las usuarias deben contar con las garantías que la producción de servicios logrará los mejores resultados que se pueda alcanzar para su situación individual y bajo estas condiciones.

4. Eficiencia. Todo establecimiento debe contar y aplicar aquellos procedimientos gerenciales que le permitan el máximo de producción al menor costo posible. De esta manera, en un contexto de linitación recurrente de recursos financieros, se puede beneficiar al mayor número posible de usuarias que requieran cuidados obstétricos esenciales. 
5. Aceptabilidad. Todo establecimiento debc conocer la cultura local, así como las necesidades y demandas de sus usuarias. De csta manera deberá seguir los procedimientos necesarios para que sus servicios no sólo respeten los valores y cultura local, sino que sean proactivos en la implementación de medidas que conduzcan a satisfacerlas, resultando atractivos para la comunidad.

Todos estos atributos interactúan entre sí, y no es posible que un scrvicio sea definido como "de calidad" si uno de csos atributos no ha sido logrado.

Evidencia de la utilidad de la mejora de calidad para reducir la mortalidad matcrna

El Proyecto 2000 ha venido trabajando intensamente con este enfoque de calidad. La cvalluación de su intervención mostró una diferencia signilicativa en la ponderación de calidad y uso de servicios en favor de los establecimientos participantes en el Proyecto, comparado con aquellos seleccionados como control. Un análisis multivariado permitió demostrar que, en aquellos establecimientos en los cuales se habia instalado procesos de mejora continua de la calidad, la cobertura de partos institucionales se incrémentó én $54 \%\left(r^{2}=0,87\right)$. Igualmente, eI análisis de las citras oliciales de muertes maternas notilicadas (1.3) por lodas las DISA del país entre 1997 y 1999 pcrmitió calcular tasas de mortalidad, utilizando escas cilias y las cstimaciones de nacidos vivos (NV) del Ministerio de Salud ( $\left.{ }^{25}\right)$. El resultado mostró que en las DISA participantes en el Proyccto 2000 la razón de mortalidad materna se redujo de $172 \times$ 100000 NV en 1997 a $127 \times 100000$ NV en el año 2000, mientras que en las DISA que no participan en el Proyecto 2000, la razón prácticamente no se modilicó (103 x $100000 \mathrm{NV}$ cn 1997 y $98 \times 00(000 \mathrm{NV}$ en 2000 )

Una mención especial debe dedicarse al caso de los departamentos de Puno y Huancavelica. Ellos forman parte de las DISA participantes en el Proyecto 2000, y el hecho de que continúan mostrando cirras de mortalidad materna muy elcvadas pareciese contradecir la alirmación que la mejora de calidad tienc un efccto positivo sobrc la mortalidad materna. Esta apreciación surge de la observación transversal de los dalos, sin apreciar las tendencias a lo largo del tiempo. El análisis de tendencias nos permite afirmar que Huancavelica redujo cl númcro de muertes notificadas de 73 en 1997 a 22 en el año 2000 , es decir, una reducción de $70 \%$, micntras que Puno lo hizo desde 109 a 79, reducción de $28 \%$ en el mismo período. La reducción del númcro de muertes notificadas de ambos departamentos está por encima del promedio nacional, que fue $15 \%$ para el período en cuestión. Evidentemente, todavía queda mucho por hacer en estos departamentos, pero, especialniente en Huancavelica, es evidente que la dirccción actual es la correcta

Los resultados de esta experiencia del $\mathrm{Mi}$ nisterio de Salud permiten afirmar que la mejora de la calidad de servicios es el camino hacia la reducción de la mortalidad matcrna y debo convertirse en la piedra angular de docerina que unilique el accionar del sistema de salud a escala nacional, adaptándola a las peculiaridades regionales.

\section{Una agenda mínina: ¿por dónde empezar?}

Construir, implementar y sostener en el tiempo una política coherente para reducir la mortalidad materna es una tarca difícil y compleja. Esto es así debido a que se requiere:

- desarrollar tecnológicamente los scrvicios de salud, con todos sus costos asociados:

- modificar drásticamente las relaciones de poder al interior de los hogares, para lavorecer la autodetcrminación de las mujeres; cntre los scrvicios de salud y la comunidad, para privilegiar cl enloque en las necesidades y denlandas de la población; y al interior de los servicios de salud, para favorecer el trabajo en cquipo;

- involucrar a un gran número de actores de naturaleza divcrsa, incluyendo organizacio- 
nes del Estado, de la socicdad civil y representantes de la comunidad;

- no cxiste consenso sobrc qué y cómo hacer para reducir la mortalidad materna; dentro del reducido ámbito del nivel central del MINSA no se ha logrado aún construir este consenso;

- para lograr un efecto significativo sobre la mortalidad materná, se requiere de un período de ticmpo prolongado, durante el cual se mantenga una misma dirección de política.

Todos estos clementos dificultan la viabilidad de una política $\left(^{26}\right)$, lo que sugiere que es necesario encontrar mecanismos para favorecer la posibilidad de implementar y sostener una polícica efectiva de reducción de la mortalidad materna. Para empezar esta difícil tarea, sc propone generar un rápido consenso sobre tres elementos clave que permitan acrecentar las posibilidades de éxito:

1. Unificar la doctrina de lucha contra la mortalidad materna alrededor del concepto de facilitar el acceso universal a servicios de salud que oferten cuidados obstétricos csenciales de calidad y promover la planificación familiar. Esta uniticación debería comprometer a organizaciones comunicarias de base, instituciones públicas, privadas, no gubernamentales, acadénicas, profesionales y politicas.

2. Focalizar, en el corto plazo, la intervención sanitaria en la mejora de la calidad de los cuidados obstétricos esenciales en los hospitales departamentales y establecimientos cabecera de red. Simultáneamente, iniciar la construcción de las redes de servicios de salud y de las capacidades institucionales y comunitarias que permitan a las mujeres rurales acceder a estos servicios de salud.

3. Modificar la estructura de los Programas Nacionales, con la linalidad de:

a. Limitar sus funciones en el nivel central a aquellas propias del planeamiento estratégico nacional y la definición, monitoreo y actualización de la normatividad técnica. b. Descentralizar las funciones operativas y de implementación a las DISA, las que adecuarán el planeamiento estratégico nacional según sus propias realidades y conducirán los procesos de mejora de calidad de los cuidados obstétricos esenciales en sus respectivos hospitales y establecimicntos cabccera de red, y construirán las redes de servicios que favorezcan el acceso de las mujeres rurales a dichos servicios.

c. Constituir los Programas Ampliados (PA), constituidos por todos los actores antes mencionados, con la linalidad de construir y sostener políticas nacionales estables. Esto implicaría que, tanto a nivel nacional como regional, la estructura actual de los Programas Nacionalcs asumiría lunciones de secretaría técnica encargada de implementar las definiciones de política de los PA.

\section{BIBLIOGRAFÍA}

1) Farr W. Anneal report of the Registrar General of England and Wales. London. 1838. Citado por: Maine D. What's sn special about maternal mortality? En: Berger M. Sundari Ravindran TK. edicores. Satc Motherhood initiacives: critical issucs. Oxford: Blackwell Science: 1999. p. 175-82.

2) Anderson J. La muerte materna en zonas rurales del perú Proyecto 2000 - MINSA. Lima; Ministerio de Salud. 1999.

3) Agarwal B, Bare TD, Henriques ML, Mathew' I, Chainery-Ilesse M, Ariffin J, Ghaid D, Jolly R, McAskie C) Stewart F. Engendering adjustments for the 1990s: Report of a Commonwealth expert group on women an structural adjustment. London: Commonwealth Secretariat: 1990.

4) Tinker A, Koblinsky MA. Hacia una maternidad segura. Documentos para discusión del Banco Mundial. Washington: Banco Mundial; 1994.

5) Instituto Nacional de Estadística e Informática. Encuesa Demográfica y de Salud Familiar 2000. Lima: INEI. 2001

6) Currey B. Maternal mortality and mothers' deadis as developinent indicacors [cartal. Br Med J 2000: $321(7264): 835$.

7) World Health Organization/UNICEF. Revistd 1990 estimates of matcrnal mortality. Ginebra: Unicer: 1996.

8) Cervantes R, Watanabe T, Denegri J. Mucrte materna y muerte perinacal en los hospitales del Perú. Lina: MINSASociedad Peruana de Ginecología y Obsterricia: 1988.

9) Kaunitz A. Spence C, Danielson TS, Rochat RW. Grimes DA. Perinatal and maternal mortality in religious group 
avoiding obsterrical care. Am J Ohstets Gynecol 1984 $150(7): 826-31$.

10) OPS/OMS. Ejecución y avances del plan de accion regionnal para la reducción de la mortalidad materna: Análisis de la situación accual. OPS. 1997.

11) Unifed Nations. UN agencies issue joint statement for reducing maternal morkalicy. 1999. Tomado de: hetp:// www. licalth. lgov. hc.

12) WHO/UNICEF. The sisterhood method for estimating maternal inortalicy: guidance notes for potential uscrs. Ginebra: Unicel; 1999.

13) Dirección General de Salud de las Personas. Mortalidad materna: buscando soluciones a un problema de salud púhlica. En: Ministerio de Salud. Salud Mujer No. 1. Lima: Ministerio de Salud: 2001. p. 13-16.

14) Oficina de Estadística e Informática-MINSA. Segundo Censo de Intraestruciura Sanitaria y Recursos del Secon Salud. Lima: Ministerio de Salud: 1996.

15) Rooks J, Weatherlyy NL, Ernst KM, Stapleton S, Rosen D, Rosenfield A. Outcomes of care in birch centers: 'The National Birth Center. N Engl J Med 1989: 321(26): 18014-11.

16) Maine D, MacCarthy J, Ward V. Guidelines for monitoring progress on the reduction of maternal mortalicy. Nueva York: UNICEF: 1992

17) Post M. Preventing maternal mortality through emergemey obsteric care. Washington: SARA Issues Papur. 1997.

18) Browne F. Antenatal care and maternal mortality. The Lancet. 1932, 2 July:1-4. Citado en: Mainc D. What's so special about maternal incirtality? En: Berger M. Sundari
Ravindran TK, edicores. Sale Modterlond initiatives: critical issues. Oxford: Blackuell Scicrec: 1999. p. 175-82.

19) Maine D. What's so special abuut maternal morality? En: Berger $M$. Sundari Ravindran TK, editores. Safi Motherhood intiatives: critical issues. Oxlord: Blackwell Science: 1999. ए. 175-82.

20) Gauderer MWL, .Iassani M.M. Izant RJ. Ultrasınograpluc antenatal diagnosis: will it change the spectrum of neonatal surgery? I Pediatr Surg 1984. Aug: 19(4):404-7.

21) Bloom SS, Lippeveld T, Wypij D. Docs antenatal carc make a difference to safe delivery? $\Lambda$ study in urban Uttar Pradesh. India. Health Policy and Planning 1999; 14(1):38-48.

22) Dierna $R$, Salazar $X$, Vargas $R$, Nacarato $P$, Guzmán C. El parto de lit vida en los andes y amazonía del Perú. Lima: Proyceto 2000 - MINSA: 1999.

23) Maxwell R. Quality assessment in healut. Br Med J 1984; 288, 6428: $1470-72$.

24) Thaddeus S. Maine D. Too far to walk: matcrnal mortality in context. Findings from a multidisciplinary literature review. Prevention of Maternal Mortalicy Program. CoIumbia: Center for Population and Fannily Health. Faculty of Medicine. Columbia University: 1990.

25) Oficina de Estadística e Informática. Estimación poblacional por departamentos y por rangos poblacionales. Lima: Miniscerjo de Salud: 2001. Disponible en: hetp:// 10.10.1.211/estadisticas. Visitado el 17 de julion de 2001.

26) Cleaves $P$. Implementation anidst scarciry and apathy: political power and policy design. 1980. Citado en: Wale G. Healeh Policy, an incroduction to process and power. Johanneshurgo: Witwatersrand Universiry Press: 1994. Págs. 168-9. 